\title{
A MODEL OF METEOROID ATMOSPHERIC ENTRY WITH IMPLICATIONS FOR THE NEO HAZARD AND THE IMPACT OF COMET SHOEMAKER-LEVY 9 ON JUPITER*
}

\author{
D. A. Crawford and M. B. Boslough \\ Sandia National Laboratories, Albuquerque, NM 87185
}

RECEIVED

APR 011996

\begin{abstract}
Summary-A new semi-analytical model describing the entry and deformation $\mathrm{A}$ S TI meteoroids entering planetary atmospheres has been developed and calibrated against numerical simulations performed using the CTH shock-physics computational hydrocode. The model starts with the classical treatment of meteoroid ablation which is modified to include an explicit treatment of energy conservation during the ablative process. This is reconciled with terrestrial observations by modeling the formation of a vapor/debris layer (the visible bolide) surrounding the central meteoroid. A mechanical deformation model based on long-wavelength hydrodynamic instability growth is added and calibrated against numerical simulations performed with CTH. The analytical model provides initial conditions for numerical fireball simulations which are compared with observations of the Comet Shoemaker-Levy 9 impact on Jupiter and can be used to assess the terrestrial impact hazard.
\end{abstract}

\section{INTRODUCTION}

In early July, 1992, periodic comet Shoemaker-Levy 9 broke up during a close encounter with Jupiter. For a brief two year period, about 20 large fragments and associated debris followed one last orbit about Jupiter before striking the planet at an estimated velocity of $60 \mathrm{~km} / \mathrm{s}$. Most of the debris collided with the planet over a 2 month period centered on July, 1994, with the largest fragments entering the Jovian atmosphere during July 16-22 (UT). The impact sites were located just beyond the limb of Jupiter and were not directly visible from Earth; nevertheless, the Galileo spacecraft was positioned for direct viewing of the impact points. Although impact phenomena were not spatially resolved by the spacecraft, its timing, spectral and luminosity data are invaluable for comparison with analytical and numerical models. Fireballs and plumes generated by the impacts were visible in line-of-sight from Earth within minutes after the more energetic impacts and the impact locations themselves rotated into view within 7-20 minutes [Hammel et al., 1995]. The wealth of data provided by this fortuitous event gives us an opportunity to assess models of meteoroid entry into planetary atmospheres. In the context of this paper, the eventual goal of our modeling effort is to provide a mechanism to assess the specific hazard associated with a large atmospheric entry event on Earth.

*This work was supported by the U.S. Dept. of Energy under contract DE-AC04-94AL85000 and funded by the laboratory directed research and development program (LDRD). 


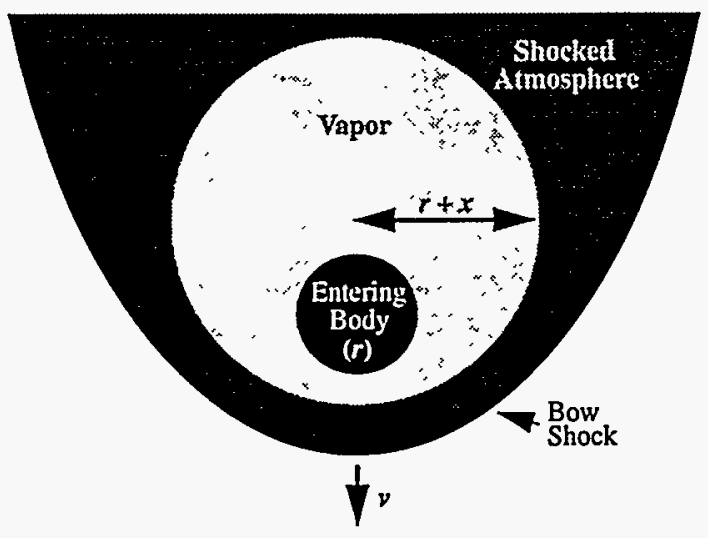

Fig. 1. Schematic diagram of the analytical entry model that explicitly models the development and behavior of an ablative vapor layer. The increased interaction cross section, $\pi(r+x)^{2}$, due to the presence of the vapor, accommodates energy conservation during the ablative process while retaining the large energy deposition rates of previous models. The thickness of the vapor layer, which changes dynamically as the impactor penetrates, is a function of impactor composition, the temperature at the bow shock and the opacity of the vapor and atmospheric gases.

\section{METEOROID ENTRY}

Understanding the mechanisms of kinetic energy loss during meteoroid traversal of planetary atmospheres is crucial for understanding the development of fireballs and plumes that were observed during the impact of Comet Shoemaker-Levy 9 on Jupiter [Crawford, 1995] and entry events in Earth's atmosphere [Boslough and Crawford, this volume]. Analytical models of the deceleration, ablation, deformation and breakup of meteoroids during passage through planetary atmospheres have been proposed and refined by many researchers [Ivanov \& Yu, 1988; Zahnle, 1992; Hills \& Goda, 1993; Ceplecha et al., 1993; Sekanina, 1994]. Here, a modified ablation model is proposed that properly satisfies conservation of energy during the ablative process and can be reconciled with observations of terrestrial meteors. A new analytical hydrodynamic deformation model, based on an observation of O'Keefe et al. (1995), compares favorably with numerical simulations performed using the CTH shock-physics code [Crawford et al., 1995]. Coupling the new hydrodynamic model to the modified ablation model yields a model that describes meteoroid entry over a wide range of velocities and spatial scales.

A body entering a planetary atmosphere at hypervelocity produces a parabolic bow shock (Fig. 1). The irreversible heating at the bow shock is radiatively coupled to the meteoroid surface causing vaporization of the meteoroid materials. Because the energy required to remove a unit mass from the surface of the body is much greater than the heat of vaporization, a vapor layer forms and thickens. The coupled differential equations that describe the evolution of the vapor layer and the deceleration and hydrodynamic deformation of the meteoroid are written as:

$$
\begin{gathered}
m \frac{d v}{d z}=\frac{C_{d}}{2} \pi(r+x)^{2} \rho v \sec \theta \\
\frac{d m}{d z}=C_{m} \pi(r+x)^{2} \rho \sec \theta \\
\frac{d x}{d z}=\frac{C_{h} R}{8 Q_{0} A} T_{v}\left(\frac{r}{r+x}\right)^{2} \sec \theta-\frac{1}{4} \frac{C_{m} R T_{v}}{A v^{2}} \sec \theta \\
\frac{d r}{d z}=k_{0}\left(\frac{\rho}{\rho_{b}}\right)^{1 / 2} \sec \theta
\end{gathered}
$$

where $m, v, r, \rho_{b}, \theta, z, Q_{0}$, and $A$ are the mass, velocity, radius, density, entry angle, altitude, heat of vaporization and average molecular weight of the entering body; $\rho$ is atmospheric density; $C_{d} \sim 1, C_{m} \sim 0.5$ and $C_{h} \sim 0.1$ are drag, mass-loss and heat-transfer coefficients; $x$ and $T_{v}$ are the 

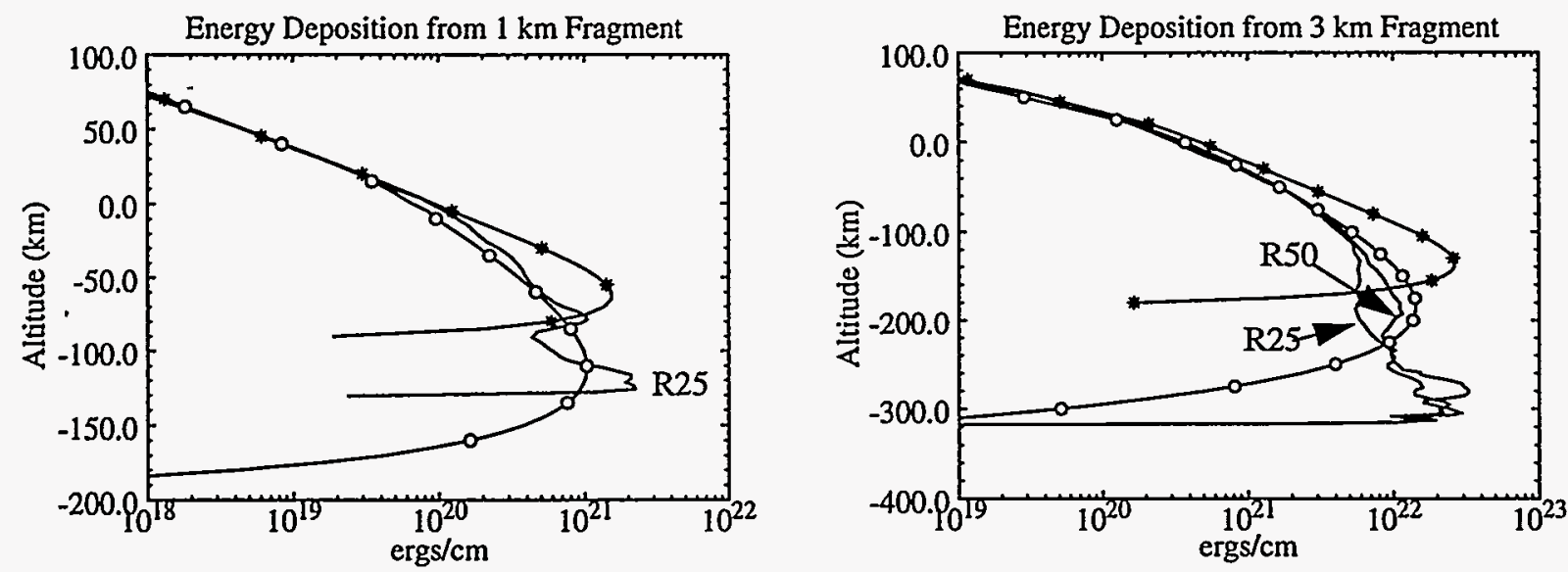

Fig. 2. Energy deposition from 1- and 3-km ice fragments entering Jupiter's atmosphere. The solid curves without symbols are from CTH simulations [9] and are labeled with the resolution of the calculation (represented by the number of zones across the radius, R25 and R50). Results of the analytical model $\left(k_{0}=0.2\right)$ are superimposed (open circles). The 'pancake' model of Zahnle and Mac Low (1994) (stars) places the peak energy deposition slightly higher.

thickness and temperature of the vapor layer; $R$ is the gas constant and $k_{0} \sim 0.2$ is a constant describing the growth rate of a Kelvin-Helmholtz instability which 'flattens' the body [Crawford, 1995].

Equations (1) - (4) are numerically integrated to describe the deceleration, ablation, vaporization and hydrodynamic deformation experienced by an entering body. The hydrodynamic deformation rate constant, $k_{0}$, is calibrated against CTH simulations (which did not model radiative ablation) by independently integrating Equations (1) and (4) and keeping $x=0$ (Fig. 2). Because the radiant energy that drives vaporization at the surface of the meteoroid must propagate through the vapor layer from the bow shock, growth of the vapor layer will stop once it is optically thick. For large objects such as Shoemaker-Levy 9, this occurs at high enough altitude to affect the dynamics at low altitude (Fig. 3). For small meteoroids, Equation (3) is self-limiting and the vapor layer will reach an equilibrium thickness (typically 5-10 times the radius of the meteoroid) before the opacity limit is reached.

Fig. 3. Energy deposition curves from the analytical model for 125-2000 m diameter ice fragments entering the Jovian atmosphere. The change of slope at an altitude of $100 \mathrm{~km}$ occurs when the vapor layer becomes optically thick and appears at about the same location as determined by Zahnle and Mac Low (1994).

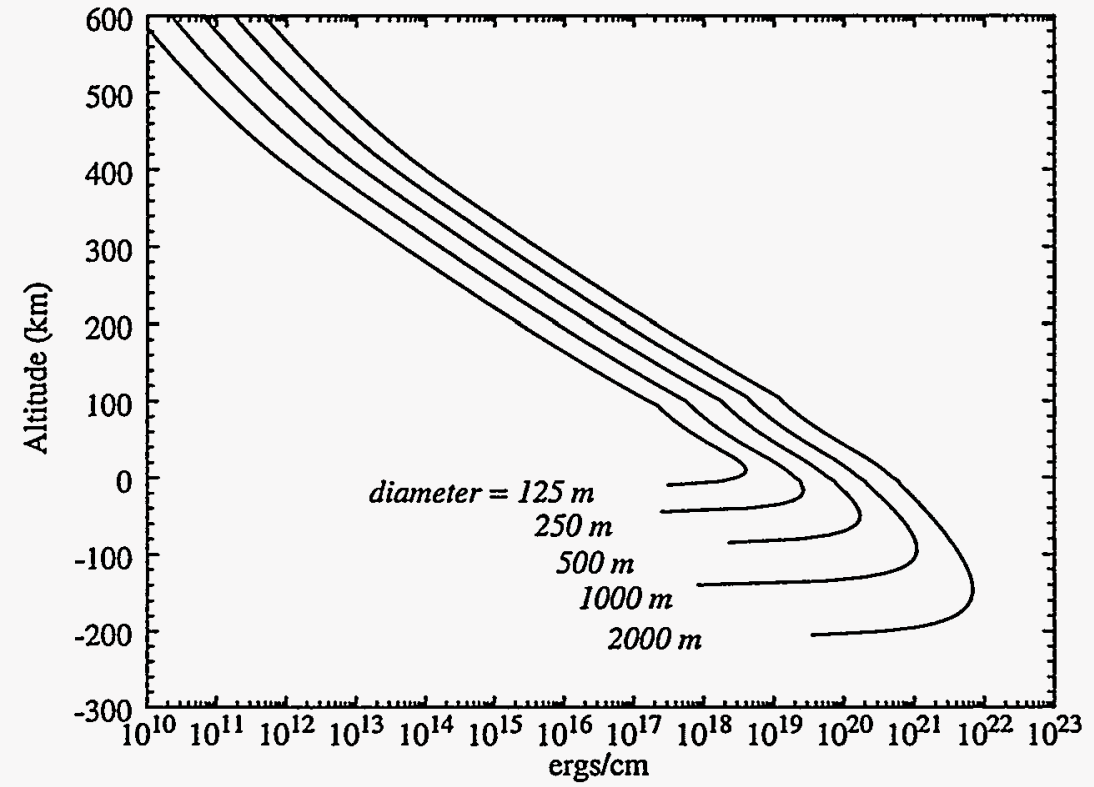


Fig. 4. Maximum altitude vs. fragment diameter for atmospheric cloud layers represented by tracer particles in numerical simulations. Eight tracer layers are shown corresponding to cloud layers originating at $11,0,-11,-21,-32$, and $-42 \mathrm{~km}$ respectively.

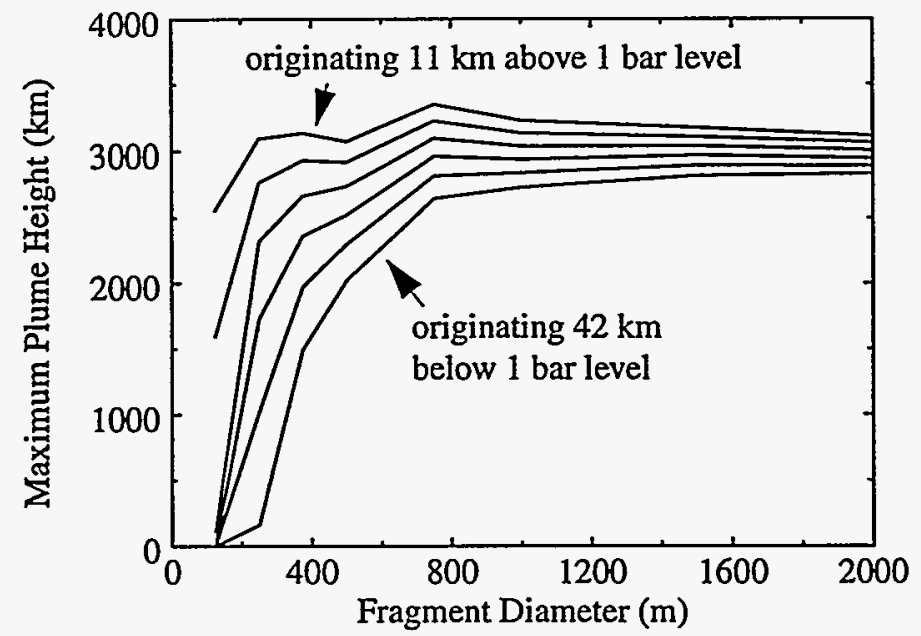

\section{PLUME EVOLUTION: COMPARISON WITH HST OBSERVATIONS}

We can use the semi-analytical meteoroid entry model as initial conditions for plume evolution models of the Shoemaker-Levy 9 event. These models are strongly constrained by the seemingly contradictory observations that plumes observed by the Hubble Space Telescope (HST) all had approximately the same maximum altitude yet the pattern of dark ejecta they left in the Jovian stratosphere varied considerably in albedo and lateral extent [Hammel et al., 1995]. Nevertheless, plume simulations using the CTH shock-physics code[McGlaun et al., 1990] and using the entry model derived in this paper for initial conditions compare well with the HST observations.

Figures 4 and 5 show results from the eight numerical fireball simulations that we have performed. A two dimensional representation, symmetric to the $45^{\circ}$ entry angle, was used to simulate the first three minutes of fireball evolution in CTH. The S-L 9 fragments were modeled as water ice with diameters of $125-2000 \mathrm{~m}$. Tracer particles were added to the simulations to represent the Jovian cloud layers (Fig. 5). At the end of the CTH simulation, the locations of the tracer particles were ballistically extrapolated in 3-D to follow plume evolution to maximum height and subsequent collapse. The plume simulations show that maximum altitude of a specific isodensity contour is an increasing function of fragment size and mass which is not consistent with the observations. If, instead, the tops of the plumes observed by HST represent a compositional transition within materials originating at a specific level of the atmosphere (an isocomposition contour), then maximum plume heights are independent of fragment size provided the fragments penetrated at least $30 \mathrm{~km}$ below this level. If the observable plumes were derived from the Jovian cloud layers, maximum plume heights of $3000 \mathrm{~km}$ are independent of fragment size (Fig. 4) and consistent with HST observations [Hammel et al., 1995]. For example, if the tops of the plumes originated from the visible cloud tops (the $\mathrm{NH}_{3}$ layer), fragment masses greater than $4 \times 10^{12} \mathrm{~g}$ (equal to $200 \mathrm{~m}$ diameter fully dense water ice) are required to explain the observations. If the visible plumes originated from the hypothetical $\mathrm{NH}_{4} \mathrm{SH}$ cloud layer, masses greater than $3 \times 10^{13} \mathrm{~g}$ ( $400 \mathrm{~m}$ water ice) are required. According to this model, the radial extent and mass density of the observable plume are functions of fragment cross-sectional area and directly relate to the radial extent and albedo of the resulting ejecta patterns. The apparent gap, seen in some of the images, between the central disturbance of the impact site and the inner front of the crescent-shaped ejecta may reflect the fragment's depth of penetration below the source of the visible ejecta. 


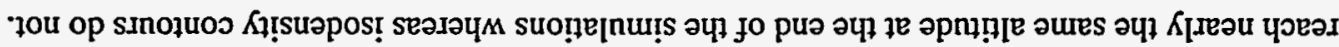

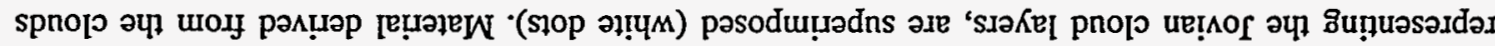

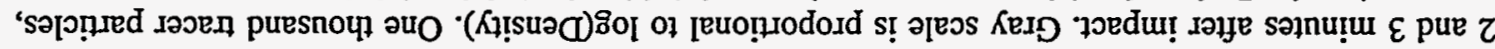

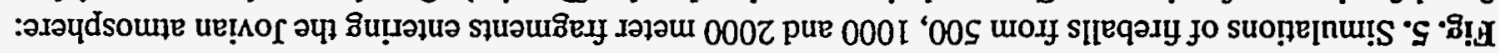

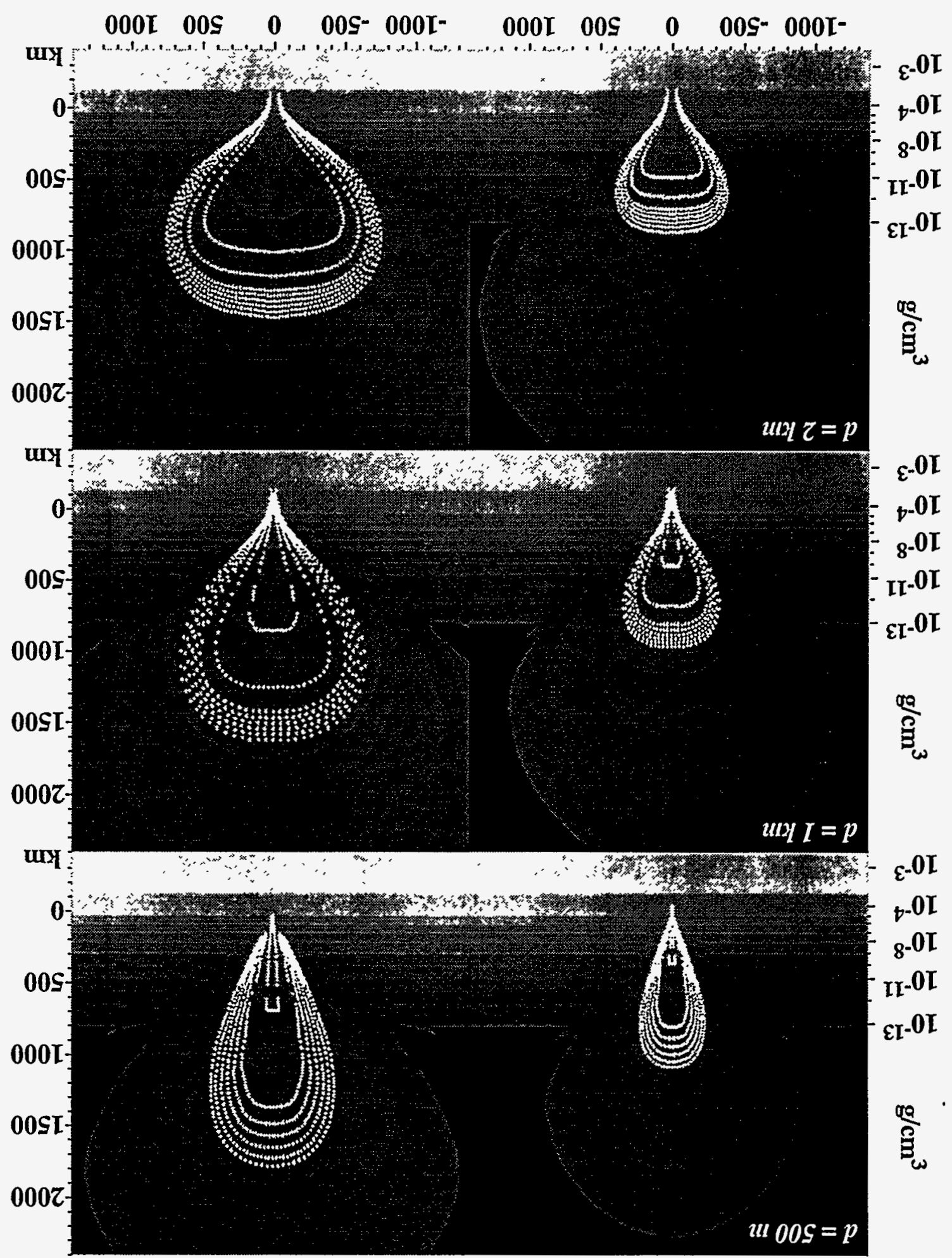




\section{CONCLUSIONS}

Previous models of radiative ablation and mass loss of bolides traversing Earth's atmosphere can be reconciled with conservation of energy provided that the dynamics of the ablative vapor layer are included. While mass loss due to radiative heating of fragments larger than $100 \mathrm{~m}$ in the terrestrial or Jovian atmospheres is insignificant, ablation is a major contributor to energy deposition at high altitude and has an important role in early-time fireball evolution. Results from fireball and plume evolution simulations using the CTH shock-physics code with initial conditions derived from the new analytical meteoroid entry model demonstrate that simulated plumes consistent with HST observations of the Shoemaker-Levy 9 impacts on Jupiter are produced as long as the tops of the plumes are made up of materials derived from Jupiter's cloud layers (rendered opaque by condensation). Using our new entry model as input for CTH simulations that match HST observations of Shoemaker-Levy 9 impacts on Jupiter provides confidence that a similar analysis can be performed for the terrestrial impact hazard (e.g. Boslough and Crawford, 1996).

\section{REFERENCES}

Boslough, M. B. and D. A. Crawford (1996), (this volume).

Ceplecha, Z. et al. (1993), Atmospheric fragmentation of meteoroids, Astron. and Astrophys., 279, 615-626.

Crawford, D. A. et al. (1995), The Impact of Periodic Comet Shoemaker-Levy 9 on Jupiter, Int. J. Impact Engin., 17, 253-262.

Crawford, D. A. (1995), Models of Fragment Penetration and Fireball Evolution, in The Collision of Comet Shoemaker-Levy 9 with Jupiter, Keith Noll, ed., Cambridge Univ. Press, (in press).

Hammel, H. B. et al. (1995), HST Imaging of Atmospheric Phenomena Created by the Impact of Comet Shoemaker-Levy 9, Science, 267, 1288-1296.

Hills, J. G. and M. P. Goda (1993), The Fragmentation of Small Asteroids in the Atmosphere, Astro. J., 105, 1114-1144. ,

Ivanov, B. A. and O. Yu (1988), Simple Hydrodynamic Model of Atmospheric Breakup of Hypervelocity Projectiles, Lun. Planet. Sci., 19, 535-536.

McGlaun, J. M. et al. (1990), CTH - A three-dimensional shock-wave physics code, Int. J. Impact Engineering, 10, 351.

O'Keefe, J. D. et al. (1994), Penetration of Large Bolides into Dense Planetary Atmospheres-Role of Hydrodynamic Instabilities, Lunar and Planetary Science, 25, 1023-1024.

Sekanina, Z. (1993), Disintegration phenomena expected during the forthcoming collision of periodic comet Shoemaker-Levy 9 with Jupiter, Science, 262, 382.

Zahnle, K. and M. M. Mac Low (1994), The Collision of Jupiter and Comet Shoemaker-Levy 9 , Icarus, 108, 1-17. 